Network Working Group

Request for Comments: 1134
D. Perkins

$\mathrm{CMU}$

November 1989

\title{
The Point-to-Point Protocol: A Proposal for Multi-Protocol Transmission of Datagrams Over Point-to-Point Links
}

\section{Table of Contents}

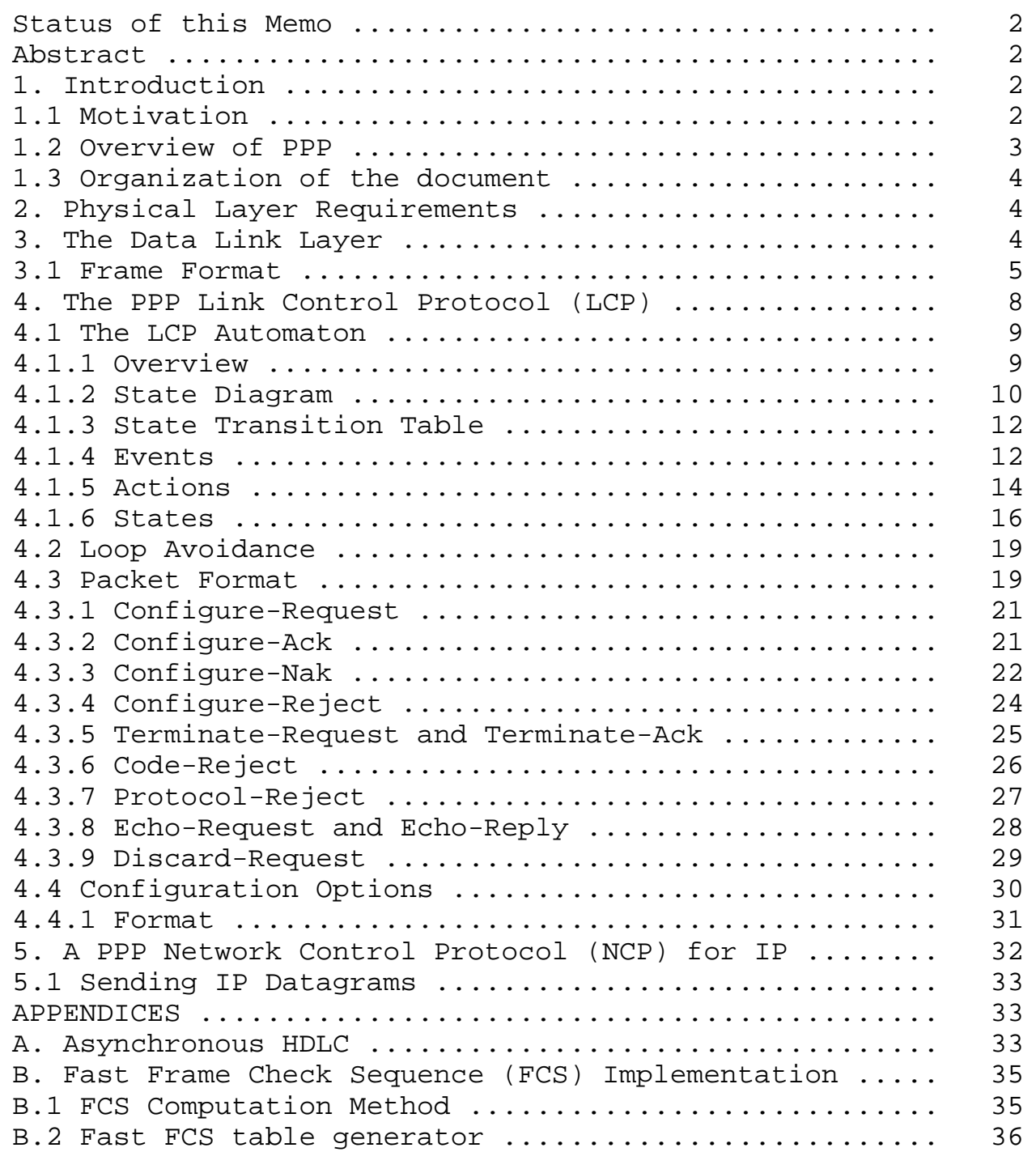




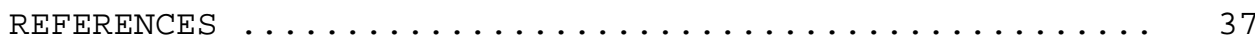

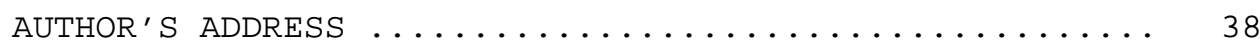

Status of this Memo

This memo defines a proposed protocol for the Internet community.

This proposal is the product of the Point-to-Point Protocol Working Group of the Internet Engineering Task Force (IETF). Comments on this memo should be submitted to the IETF Point-to-Point Protocol Working Group chair by January 15, 1990. Comments will be reviewed at the February 1990 IETF meeting, with the goal of advancing PPP to draft standard status. Distribution of this memo is unlimited.

Abstract

The Point-to-Point Protocol (PPP) provides a method for transmitting datagrams over serial point-to-point links. PPP is composed of three parts:

1. A method for encapsulating datagrams over serial links.

2. An extensible Link Control Protocol (LCP).

3. A family of Network Control Protocols (NCP) for establishing and configuring different network-layer protocols.

This document defines the encapsulation scheme, the basic LCP, and an NCP for establishing and configuring the Internet Protocol (IP) (called the IP Control Protocol, IPCP).

The options and facilities used by the LCP and the IPCP are defined in separate documents. Control protocols for configuring and utilizing other network-layer protocols besides IP (e.g., DECNET, OSI) are expected to be developed as needed.

1. Introduction

\subsection{Motivation}

In the last few years, the Internet has seen explosive growth in the number of hosts supporting TCP/IP. The vast majority of these hosts are connected to Local Area Networks (LANs) of various types, Ethernet being the most common. Most of the other hosts are connected through Wide Area Networks (WANs) such as X.25 style Public Data Networks (PDNs). Relatively few of these hosts are connected with simple point-to-point (i.e., serial) links. Yet, point-to-point links are among the oldest methods of data communications and almost 
every host supports point-to-point connections. For example, asynchronous RS-232-C [1] interfaces are essentially ubiquitous.

One reason for the small number of point-to-point IP links is the lack of a standard encapsulation protocol. There are plenty of nonstandard (and at least one defacto standard) encapsulation protocols available, but there is not one which has been agreed upon as an Internet Standard. By contrast, standard encapsulation schemes do exist for the transmission of datagrams over most popular LANs.

One purpose of this memo is to remedy this problem. But even more importantly, the Point-to-Point Protocol proposes more than just an encapsulation scheme. Point-to-Point links tend to exacerbate many problems with the current family of network protocols. For instance, assignment and management of IP addresses, which is a problem even in LAN environments, is especially difficult over switched point-topoint circuits (e.g., dialups).

Some additional issues addressed by PPP include asynchronous (start/stop) and bit-oriented synchronous encapsulation, network protocol multiplexing, link configuration, link quality testing, error detection, and option negotiation for such capabilities as network-layer address negotiation and data compression negotiation.

PPP addresses these issues by providing an extensible Link Control Protocol (LCP) and a family of Network Control Protocols (NCP) to negotiate optional configuration parameters and facilities.

\subsection{Overview of PPP}

PPP has three main components:

1. A method for encapsulating datagrams over serial links. PPP uses HDLC as a basis for encapsulating datagrams over pointto-point links.

2. An extensible Link Control Protocol (LCP) to establish, configure, and test the data-link connection.

3. A family of Network Control Protocols (NCP) for establishing and configuring different network-layer protocols. PPP is designed to allow the simultaneous use of multiple networklayer protocols.

In order to establish communications over a point-to-point link, the originating PPP would first send LCP packets to configure and test the data link. After the link has been establish and optional facilities have been negotiated as needed by the LCP, the originating 
PPP would send NCP packets to choose and configure one or more network-layer protocols. Once each of the chosen network-layer protocols has been configured, datagrams from each network-layer protocol can be sent over the link.

The link will remain configured for communications until explicit LCP or NCP packets close the link down, or until some external event occurs (e.g., inactivity timer expires or user intervention).

\subsection{Organization of the document}

This memo is divided into several sections. Section 2 discusses the physical-layer requirements of PPP. Section 3 describes the Data Link Layer including the PPP frame format and data link encapsulation scheme. Section 4 specifies the LCP including the connection establishment and option negotiation procedures. Section 5 specifies the IP Control Protocol (IPCP), which is the NCP for the Internet Protocol, and describes the encapsulation of IP datagrams within PPP packets. Appendix A summarizes important features of asynchronous HDLC, and Appendix B describes an efficient table-lookup algorithm for fast Frame Check Sequence (FCS) computation.

2. Physical Layer Requirements

The Point-to-Point Protocol is capable of operating across any DTE/DCE interface (e.g., EIA RS-232-C, EIA RS-422, EIA RS-423 and CCITT V.35). The only absolute requirement imposed by PPP is the provision of a duplex circuit, either dedicated or switched, which can operate in either an asynchronous (start/stop) or synchronous bit-serial mode, transparent to PPP Data Link Layer frames. PPP does not impose any restrictions regarding transmission rate, other than those imposed by the particular DTE/DCE interface in use.

PPP does not require the use of modem control signals, such as Request To Send (RTS), Clear To Send (CTS), Data Carrier Detect (DCD), and Data Terminal Ready (DTR). However, using such signals when available can allow greater functionality and performance.

3. The Data Link Layer

The Point-to-Point Protocol uses the principles, terminology, and frame structure of the International Organization For Standardization's (ISO) High-level Data Link Control (HDLC) procedures (ISO 3309-1979 [2]), as modified by ISO 3309:1984/PDAD1 "Addendum 1: Start/stop transmission" [5]. ISO 3309-1979 specifies the HDLC frame structure for use in synchronous environments. ISO 3309:1984/PDAD1 specifies proposed modifications to ISO 3309-1979 to allow its use in asynchronous environments. 
The PPP control procedures use the definitions and Control field encodings standardized in ISO 4335-1979 [3] and ISO 43351979/Addendum 1-1979 [4]. The PPP frame structure is also consistent with CCITT Recommendation X.25 LAPB [6], since that too is based on HDLC.

Note: ISO 3309:1984/PDAD1 is a Proposed Draft standard. At present, it seems that ISO 3309:1984/PDAD1 is stable and likely to become an International standard. Therefore, we feel comfortable about using it before it becomes an International standard. The progress of this proposal should be tracked and encouraged by the Internet community.

The purpose of this memo is not to document what is already standardized in ISO 3309. We assume that the reader is already familiar with HDLC, or has access to a copy of [2] or [6]. Instead, this paper attempts to give a concise summary and point out specific options and features used by PPP. Since "Addendum 1: Start/stop transmission", is not yet standardized and widely available, it is summarized in Appendix A.

\subsection{Frame Format}

A summary of the standard PPP frame structure is shown below. The fields are transmitted from left to right.

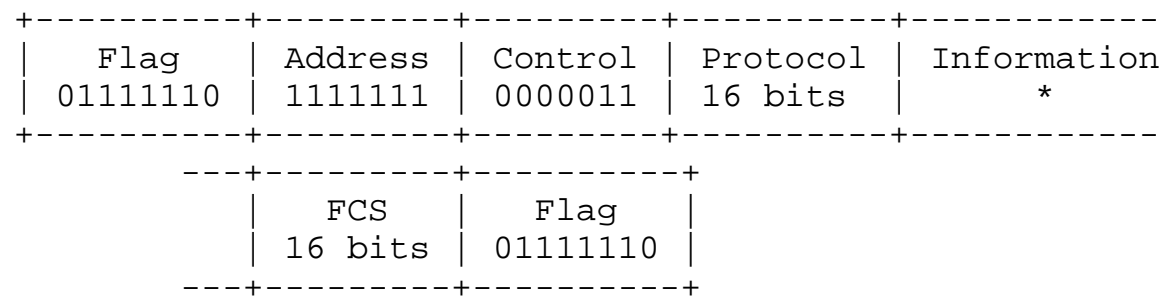

This figure does not include start/stop bits (for asynchronous links) or any bits or octets inserted for transparency. When asynchronous links are used, all octets are transmitted with one start bit, eight bits of data, and one stop bit. There is no provision in either PPP or ISO 3309:1984/PDAD1 for seven bit asynchronous links.

To remain consistent with standard Internet practice, and avoid confusion for people used to reading RFCs, all binary numbers in the following descriptions are in Most Significant Bit to Least Significant Bit order, reading from left to right, unless otherwise indicated. Note that this is contrary to standard ISO and CCITT practice which orders bits as transmitted (i.e., network bit order). Keep this in mind when comparing this document with the international 
standards documents.

Flag Sequence

The Flag Sequence is a single octet and indicates the beginning or end of a frame. The Flag sequence consists of the binary sequence 01111110 (hexadecimal 0x7e).

Address Field

The Address field is a single octet and contains the binary sequence 11111111 (hexadecimal 0xff), the All-Stations address. PPP does not assign individual station addresses. The AllStations address should always be recognized and received. Frames with other Addresses should be silently discarded.

Control Field

The Control field is a single octet and contains the binary sequence 00000011 (hexadecimal 0x03), the Unnumbered Information (UI) command with the $\mathrm{P} / \mathrm{F}$ bit is set to zero. Frames with other Control field values should be silently discarded.

Protocol Field

The Protocol field is two octets and its value identifies the protocol encapsulated in the Information field of the frame. The most up-to-date values of the Protocol field are specified in the most recent "Assigned Numbers" RFC [11]. Initial values are also listed below.

Protocol field values in the "cxxx" range identify datagrams as belonging to the Link Control Protocol (LCP) or associated protocols. Values in the "8xxx" range identify datagrams belonging to the family of Network Control Protocols (NCP). Values in the "Oxxx" range identify the network protocol of specific datagrams.

This Protocol field is defined by PPP and is not a field defined by HDLC. However, the Protocol field is consistent with the ISO 3309 extension mechanism for Address fields. All Protocols MUST be odd; the least significant bit of the least significant octet MUST equal "1". Also, all Protocols MUST be assigned such that the least significant bit of the most significant octet equals "0". Frames received which don't comply with these rules should be considered as having an unrecognized Protocol, and should be handled as specified by the LCP. The Protocol field is transmitted and received most significant octet first. 
The Protocol field is initially assigned as follows:

Value (in hex
0001 to $001 f$
0021
0023
0025
0027
0029
$002 b$
$002 d$
$002 f$
8021
8023
8025
8027
8029
$802 b$
$802 d$
$802 f$
$c 021$
$c 023$

Protocol

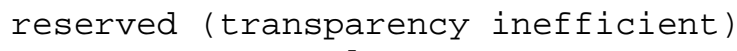


bit (four-octet) FCS for improved error detection.

The FCS field is calculated over all bits of the Address, Control, Protocol and Information fields not including any start and stop bits (asynchronous) and any bits (synchronous) or octets (asynchronous) inserted for transparency. This does not include the Flag Sequences or FCS field. The FCS is transmitted with the coefficient of the highest term first.

For more information on the specification of the FCS, see ISO 3309 or CCITT X.25.

Note: A fast, table-driven implementation of the 16-bit FCS algorithm is shown in Appendix B. This implementation is based on [7] and [8].

Modifications to the Basic Frame Format

The Link Control Protocol can negotiate modifications to the standard PPP frame structure. However, modified frames will always be clearly distinguishable from standard frames.

4. The PPP Link Control Protocol (LCP)

The Link Control Protocol (LCP) provides a method of establishing, configuring, maintaining and terminating the point-to-point connection. LCP goes through four distinct phases:

Phase 1: Link Establishment and Configuration Negotiation

Before any network-layer datagrams (e.g., IP) may be exchanged, LCP must first open the connection through an exchange of Configure packets. This exchange is complete, and the Open state entered, once a Configure-Ack packet (described below) has been both sent and received. Any non-LCP packets received before this exchange is complete are silently discarded.

It is important to note that LCP handles configuration only of the link; LCP does not handle configuration of individual networklayer protocols. In particular, all Configuration Parameters which are independent of particular network-layer protocols are configured by LCP. All Configuration Options are assumed to be at default values unless altered by the configuration exchange.

Phase 2: Link Quality Determination

LCP allows an optional Link Quality Determination phase following transition to the LCP Open state. In this phase, the link is 
tested to determine if the link quality is sufficient to bring up network-layer protocols. This phase is completely optional. LCP may delay transmission of network-layer protocol information until this phase is completed.

The procedure for Link Quality Determination is unspecified and may vary from implementation to implementation, or because of user-configured parameters, but only so long as the procedure doesn't violate other aspects of LCP. One suggested method is to use LCP Echo-Request and Echo-Reply packets.

What is important is that this phase may persist for any length of time. Therefore, implementations should avoid fixed timeouts when waiting for their peers to advance to phase 3 .

Phase 3: Network-Layer Protocol Configuration Negotiation

Once LCP has finished the Link Quality Determination phase, network-layer protocols may be separately configured by the appropriate Network Control Protocols (NCP), and may be brought up and taken down at any time. If LCP closes the link, it informs the network-layer protocols so that they may take appropriate action.

Phase 4: Link Termination

LCP may terminate the link at any time. This will usually be done at the request of a human user, but may happen because of a physical event such as the loss of carrier, or the expiration of an idle-period timer.

4.1. The LCP Automation

\subsubsection{Overview}

LCP is specified by a number of packet formats and a finite-state automation. This section presents an overview of the LCP automation, followed by a representation of it as both a state diagram and a state transition table.

There are three classes of LCP packets:

1. Link Establishment packets used to establish and configure a link, (e.g., Configure-Request, Configure-Ack, Configure-Nak and Configure-Reject)

2. Link Termination packets used to terminate a link, (e.g., Terminate-Request and Terminate-Ack) 
3. Link Maintenance packets used to manage and debug a link, (e.g., Code-Reject, Protocol-Reject, Echo-Request, Echo-Reply and Discard-Request)

The finite-state automation is defined by events, state transitions and actions. Events include receipt of external commands such as Open and Close, expiration of the Restart timer, and receipt of packets from a LCP peer. Actions include the starting of the Restart timer and transmission of packets.

\subsubsection{State Diagram}

The state diagram which follows describes the sequence of events for reaching agreement on Configuration Options (opening the PPP connection) and for later closing of the connection. The state machine is initially in the closed state (1). Once the Open state (6) has been reached, both ends of the link have met the requirement of having both sent and received a Configure-Ack packet.

In the state diagram, events are shown above horizontal lines. Actions are shown below horizontal lines. Two types of LCP packets Configure-Naks and Configure-Rejects - are not differentiated in the state diagram. As will be described later, these packets do indeed serve different, though similar, functions. However, at the level of detail of this state diagram, they always cause the same transition.

Since a more detailed specification of the LCP automation is given in a state transition table in the following section, implementation should be done by consulting it rather than this state diagram. 


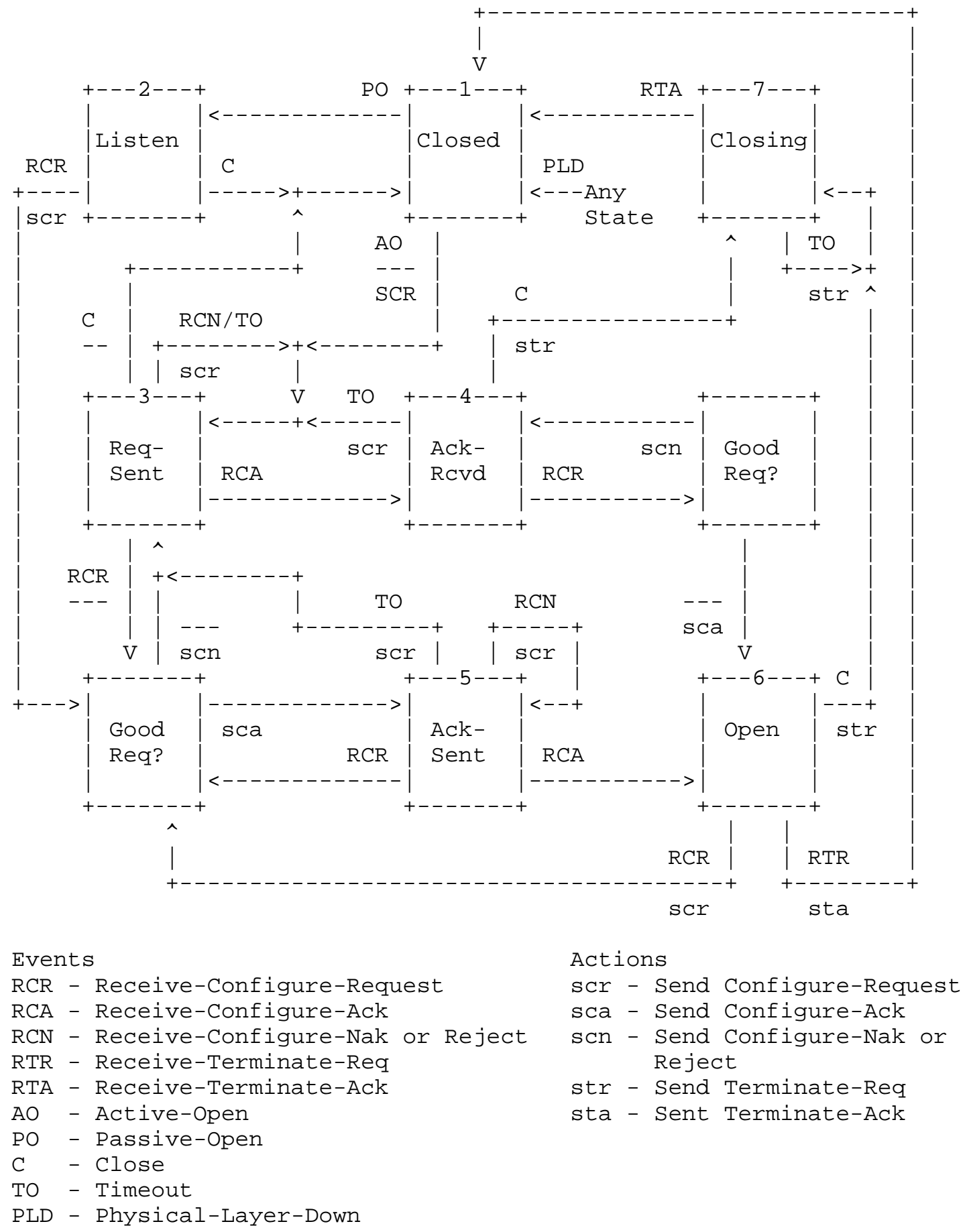




\subsubsection{State Transition Table}

The complete state transition table follows. States are indicated horizontally, and events are read vertically. State transitions and actions are represented in the form action/new-state. Two actions caused by the same event are represented as action1\&action2.

\begin{tabular}{|c|c|c|c|c|c|c|c|}
\hline Events & $\begin{array}{c}\text { State } \\
1 \\
\text { Closed }\end{array}$ & $\begin{array}{c}2 \\
\text { Listen }\end{array}$ & $\begin{array}{c}3 \\
\text { Req-Sent }\end{array}$ & $\begin{array}{c}4 \\
\mathrm{Ack}-\mathrm{RcVd}\end{array}$ & $\begin{array}{c}5 \\
\mathrm{Ack}-\text { Sent }\end{array}$ & $\begin{array}{c}6 \\
\text { Open }\end{array}$ & $\begin{array}{c}7 \\
\text { Closing }\end{array}$ \\
\hline \multicolumn{8}{|c|}{------+-------------------------------------------------------------1} \\
\hline $\mathrm{AO}$ & $\mathrm{scr} / 3$ & $\operatorname{scr} / 3$ & 3 & 4 & 5 & 6 & $\mathrm{scr} / 3$ \\
\hline $\mathrm{PO}$ & 2 & 2 & $2 *$ & 4 & 5 & 6 & sta/3* \\
\hline $\mathrm{C}$ & 1 & 1 & $1 *$ & 1 & str $/ 7$ & str $/ 7$ & 7 \\
\hline TO & 1 & 2 & $\operatorname{scr} / 3$ & $\operatorname{scr} / 3$ & $\operatorname{scr} / 3$ & 6 & str/7* \\
\hline PLD & 1 & 1 & 1 & 1 & 1 & 1 & 1 \\
\hline $\mathrm{RCR}+$ & sta/1 & scr\&sca/ 5 & $\mathrm{sca} / 5$ & $\mathrm{sca} / 6$ & $\mathrm{sca} / 5$ & scr\&sca/ 5 & 7 \\
\hline $\mathrm{RCR}-$ & sta/1 & $\operatorname{scr} \& \operatorname{scn} / 3$ & $\mathrm{scn} / 3$ & $\mathrm{scn} / 4$ & $\mathrm{scn} / 3$ & $\operatorname{scr} \& \mathrm{scn} / 3$ & 7 \\
\hline $\mathrm{RCA}$ & sta/1 & sta/2 & 4 & $\operatorname{scr} / 3$ & 6 & $\operatorname{scr} / 3$ & 7 \\
\hline $\mathrm{RCN}$ & sta/1 & sta/2 & $\operatorname{scr} / 3$ & $\operatorname{scr} / 3$ & $\operatorname{scr} / 5$ & $\operatorname{scr} / 3$ & 7 \\
\hline RTR & sta/1 & sta/2 & sta/3 & sta/3 & sta/3 & sta/1 & sta/7 \\
\hline $\mathrm{RTA}$ & 1 & 2 & 3 & 3 & 3 & 1 & 1 \\
\hline $\mathrm{RCJ}$ & 1 & 2 & 1 & 1 & 1 & 1 & 1 \\
\hline RUC & $\mathrm{scj} / 1$ & $\mathrm{scj} / 1$ & $\mathrm{scj} / 1$ & $\mathrm{scj} / 1$ & $\mathrm{scj} / 1$ & $\mathrm{scj} / 1$ & $1 \mathrm{scj} / 1$ \\
\hline RER & sta/1 & sta/2 & 3 & 4 & 5 & $\operatorname{ser} / 1$ & 7 \\
\hline
\end{tabular}

Notes:

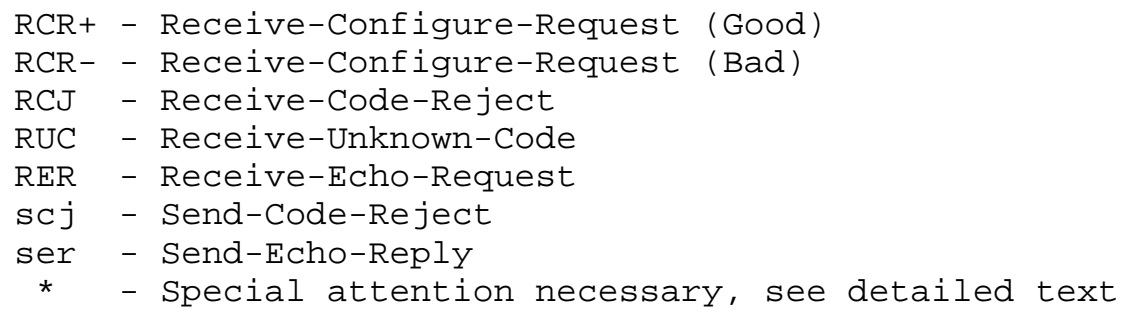

\subsubsection{Events}

Transitions and actions in the LCP state machine are caused by events. Some events are caused by commands executed at the local end (e.g., Active-Open, Passive-Open, and Close), others are caused by the receipt of packets from the remote end (e.g., Receive- ConfigureRequest, Receive-Configure-Ack, Receive-Configure-Nak, ReceiveTerminate-Request and Receive-Terminate-Ack), and still others are caused by the expiration of the Restart timer started as the result of other events (e.g., Timeout).

Following is a list of LCP events. 
Active-Open (AO)

The Active-Open event indicates the local execution of an ActiveOpen command by the network administrator (human or program). When this event occurs, LCP should immediately attempt to open the connection by exchanging configuration packets with the LCP peer.

Passive-Open (PO)

The Passive-Open event is similar to the Active-Open event. However, instead of immediately exchanging configuration packets, LCP should wait for the peer to send the first packet. This will only happen after an Active-Open event in the LCP peer.

Close (C)

The Close event indicates the local execution of a close command. When this event occurs, LCP should immediately attempt to close the connection.

Timeout (TO)

The Timeout event indicates the expiration of the LCP Restart timer. The LCP Restart timer is started as the result of other LCP events.

The Restart timer is used to time out transmissions of ConfigureRequest and Terminate-Request packets. Expiration of the Restart timer causes a Timeout event, which triggers the corresponding Configure-Request or Terminate-Request packet to be retransmitted. The Restart timer MUST be configurable, but should default to three (3) seconds.

Receive-Configure-Request (RCR)

The Receive-Configure-Request event occurs when a ConfigureRequest packet is received from the LCP peer. The ConfigureRequest packet indicates the desire to open a LCP connection and may specify Configuration Options. The Configure-Request packet is more fully described in a later section.

Receive-Configure-Ack (RCA)

The Receive-Configure-Ack event occurs when a valid Configure-Ack packet is received from the LCP peer. The Configure-Ack packet is a positive response to a Configure-Request packet. 
Receive-Configure-Nak (RCN)

The Receive-Configure-Nak event occurs when a valid Configure-Nak or Configure-Reject packet is received from the LCP peer. The Configure-Nak and Configure-Reject packets are negative responses to a Configure-Request packet.

Receive-Terminate-Request (RTR)

The Receive-Terminate-Request event occurs when a TerminateRequest packet is received from the LCP peer. The TerminateRequest packet indicates the desire to close the LCP connection.

Receive-Terminate-Ack (RTA)

The Receive-Terminate-Ack event occurs when a Terminate-Ack packet is received from the LCP peer. The Terminate-Ack packet is a response to a Terminate-Request packet.

Receive-Code-Reject (RCJ)

The Receive-Code-Reject event occurs when a Code-Reject packet is received from the LCP peer. The code-Reject packet communicates an error that immediately closes the connection.

Receive-Unknown-Code (RUC)

The Receive-Unknown-Code event occurs when an un-interpretable packet is received from the LCP peer. The Code-Reject packet is a response to an unknown packet.

Receive-Echo-Request (RER)

The Receive-Echo-Request event occurs when a Echo-Request, EchoReply, or Discard-Request packet is received from the LCP peer. The Echo-Reply packet is a response to a Echo-Request packet. There is no reply to a Discard-Request.

Physical-Layer-Down (PLD)

The Physical-Layer-Down event occurs when the Physical Layer indicates that it is down.

\subsubsection{Actions}

Actions in the LCP state machine are caused by events and typically indicate the transmission of packets and/or the starting or stopping of the Restart timer. Following is a list of LCP actions. 
Send-Configure-Request (scr)

The Send-Configure-Request action transmits a Configure-Request packet. This indicates the desire to open a LCP connection with a specified set of Configuration Options. The Restart timer is started after the Configure-Request packet is transmitted, to guard against packet loss.

Send-Configure-Ack (sca)

The Send-Configure-Ack action transmits a Configure-Ack packet. This acknowledges the receipt of a Configure-Request packet with an acceptable set of Configuration Options.

Send-Configure-Nak (scn)

The Send-Configure-Nak action transmits a Configure-Nak or Configure-Reject packet, as appropriate. This negative response reports the receipt of a Configure-Request packet with an unacceptable set of Configuration Options. Configure-Nak packets are used to refuse a Configuration option value, and to suggest a new, acceptable value. Configure-Reject packets are used to refuse all negotiation about a Configuration Option, typically because it is not recognized or implemented. The use of Configure-Nak vs. Configure-Reject is more fully described in the section on LCP Packet Formats.

Send-Terminate-Req (str)

The Send-Terminate-Request action transmits a Terminate-Request packet. This indicates the desire to close a LCP connection. The Restart timer is started after the Terminate-Request packet is transmitted, to guard against packet loss.

Send-Terminate-Ack (sta)

The Send-Terminate-Request action transmits a Terminate-Ack packet. This acknowledges the receipt of a Terminate-Request packet or otherwise confirms the belief that a LCP connection is Closed.

Send-Code-Reject (scj)

The Send-Code-Reject action transmits a Code-Reject packet. This indicates the receipt of an unknown type of packet. This is an unrecoverable error which causes immediate transitions to the closed state on both ends of the link. 
Send-Echo-Reply (ser)

The Send-Echo-Reply action transmits an Echo-Reply packet. This acknowledges the receipt of an Echo-Request packet.

4.1.6. States

Following is a more detailed description of each LCP state.

Closed (1)

The initial and final state is the closed state. In the Closed state the connection is down and there is no attempt to open it; all connection requests from peers are rejected. Physical-LayerDown events always cause an immediate transition to the closed state.

There are two events which cause a transition out of the closed state, Active-Open and Passive-Open. Upon an Active-Open event, a Configure-Request is transmitted, the Restart timer is started, and the Request-Sent state is entered. Upon a Passive-Open event, the Listen state is entered immediately. Upon receipt of any packet, with the exception of a Terminate-Ack, a Terminate-Ack is sent. Terminate-Acks are silently discarded to avoid creating a loop.

The Restart timer is not running in the closed state. The Physical Layer connection may be disconnected at any time when in the LCP Closed state.

Listen (2)

The Listen state is similar to the closed state in that the connection is down and there is no attempt to open it. However, peer connection requests are no longer rejected.

Upon receipt of a Configure-Request, a Configure-Request is immediately transmitted and the Restart timer is started. The received Configuration Options are examined and the proper response is sent. If a Configure-Ack is sent, the Ack-Sent state is entered. Otherwise, if a Configure-Nak or Configure-Reject is sent, the Request-Sent state is entered. In either case, LCP exits its passive state, and begins to actively open the connection. Terminate-Ack packets are sent in response to either Configure-Ack or Configure-Nak packets,

The Restart timer is not running in the Listen state. 
Request-Sent (3)

In the Request-Sent state an active attempt is made to open the connection. A Configure-Request has been sent and the Restart timer is running, but a Configure-Ack has not yet been received nor has one been sent.

Upon receipt of a Configure-Ack, the Ack-Received state is immediately entered. Upon receipt of a Configure-Nak or Configure-Reject, the Configure-Request Configuration Options are adjusted appropriately, a new Configure-Request is transmitted, and the Restart timer is restarted. Similarly, upon the expiration of the Restart timer, a new Configure-Request is transmitted and the Restart timer is restarted. Upon receipt of a Configure-Request, the Configuration Options are examined and if acceptable, a Configure-Ack is sent and the Ack-Sent state is entered. If the Configuration Options are unacceptable, a Configure-Nak or Configure-Reject is sent as appropriate.

Since there is an outstanding Configure-Request in the RequestSent state, special care must be taken to implement the PassiveOpen and Close events; otherwise, it is possible for the LCP peer to think the connection is open. Processing of either event should be postponed until there is reasonable assurance that the peer is not open. In particular, the Restart timer should be allowed to expire.

Ack-Received (4)

In the Ack-Received state, a Configure-Request has been sent and a Configure-Ack has been received. The Restart timer is still running since a Configure-Ack has not yet been transmitted.

Upon receipt of a Configure-Request with acceptable Configuration Options, a Configure-Ack is transmitted, the Restart timer is stopped and the Open state is entered. If the Configuration Options are unacceptable, a Configure-Nak or Configure-Reject is sent as appropriate. Upon the expiration of the Restart timer, a new Configure-Request is transmitted, the Restart timer is restarted, and the state machine returns to the Request-Sent state.

Ack-Sent (5)

In the Ack-Sent state, a Configure-Ack and a Configure-Request have been sent but a Configure-Ack has not yet been received. The Restart timer is always running in the Ack-Sent state. 
Upon receipt of a Configure-Ack, the Restart timer is stopped and the Open state is entered. Upon receipt of a Configure-Nak or Configure-Reject, the Configure-Request Configuration Options are adjusted appropriately, a new Configure-Request is transmitted, and the Restart timer is restarted. Upon the expiration of the Restart timer, a new Configure-Request is transmitted, the Restart timer is restarted, and the state machine returns to the Requestsent state.

Open (6)

In the Open state, a connection exists and data may be communicated over the link. The Restart timer is not running in the Open state.

In normal operation, only two events cause transitions out of the Open state. Upon receipt of a Close command, a Terminate-Request is transmitted, the Restart timer is started, and the closing state is entered. Upon receipt of a Terminate-Request, a Terminate-Ack is transmitted and the Closed state is entered. Upon receipt of an Echo-Request, an Echo-Reply is transmitted. Similarly, Echo-Reply and Discard-Request packets are silently discarded or processed as expected. All other events cause immediate transitions out of the Open state and should be handled as if the state machine were in the Listen state.

Closing (7)

In the closing state, an active attempt is made to close the connection. A Terminate-Request has been sent and the Restart timer is running, but a Terminate-Ack has not yet been received.

Upon receipt of a Terminate-Ack, the Closed state is immediately entered. Upon the expiration of the Restart timer, a new Terminate-Request is transmitted and the Restart timer is restarted. After the Restart timer has expired Max-Restart times, this action may be skipped, and the Closed state may be entered. Max-Restart MUST be a configurable parameter.

Since there is an outstanding Terminate-Request in the closing state, special care must be taken to implement the Passive-Open event; otherwise, it is possible for the LCP peer to think the connection is open. Processing of the Passive-Open event should be postponed until there is reasonable assurance that the peer is not open. In particular, the implementation should wait until the state machine would normally transition to the closed state because of a Receive-Terminate-Ack event or Max-Restart Timeout events. 


\subsection{Loop Avoidance}

Note that the protocol makes a reasonable attempt at avoiding Configuration Option negotiation loops. However, the protocol does NOT guarantee that loops will not happen. As with any negotiation, it is possible to configure two PPP implementations with conflicting policies that will never converge. It is also possible to configure policies which do converge, but which take significant time to do so. Implementors should keep this in mind and should implement loop detection mechanisms or higher level timeouts. If a timeout is implemented, it MUST be configurable.

For example, implementations could take care to avoid ConfigureRequest or Terminate-Request livelocks by using a Max-Retries counter. A Configure-Request livelock could occur when an originating $\mathrm{PPP}$ sends and re-sends a $\mathrm{C}-\mathrm{R}$ without receiving a reply (e.g., the receiving $\mathrm{PPP}$ entity may have died). A Terminate-Request livelock could occur when the originating PPP sends and re-sends a $\mathrm{T}-\mathrm{R}$ without receiving a Terminate-Ack (e.g., the $\mathrm{T}-\mathrm{A}$ may have been lost, but the remote PPP may have already terminated). Max-Retries indicates the number of packet retransmissions that are allowed before there is reasonable assurance that a livelock situation exists. Max-Retries MUST also be configurable, but should default to ten (10) retransmissions.

\subsection{Packet Format}

Exactly one Link Control Protocol packet is encapsulated in the Information field of PPP Data Link Layer frames where the Protocol field indicates type hex c021 (Link Control Protocol).

A summary of the Link Control Protocol packet format is shown below. The fields are transmitted from left to right.

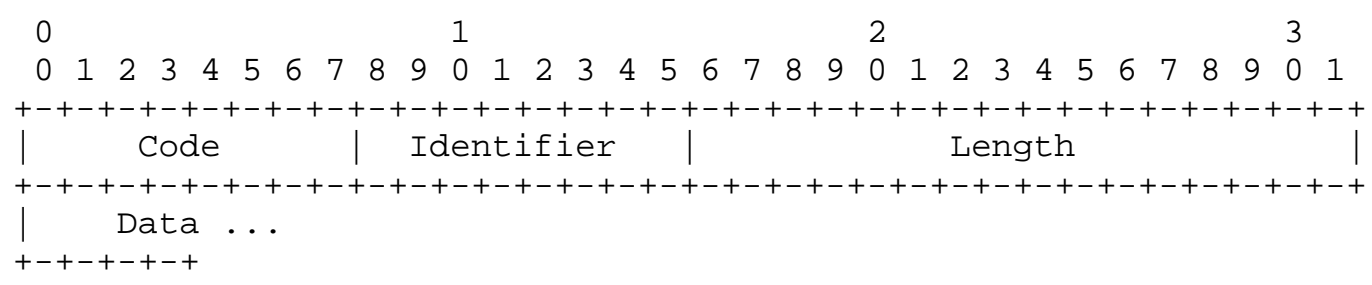

Code

The code field is one octet and identifies the kind of LCP packet. LCP Codes are assigned as follows: 


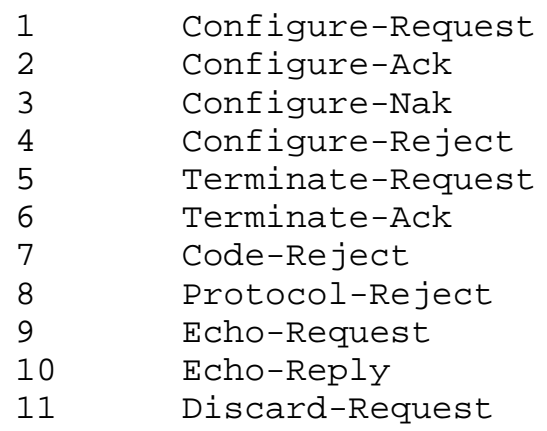

Identifier

The Identifier field is one octet and aids in matching requests and replies.

Length

The Length field is two octets and indicates the length of the LCP packet including the Code, Identifier, Length and Data fields. Octets outside the range of the Length field should be treated as Data Link Layer padding and should be ignored on reception.

Data

The Data field is zero or more octets as indicated by the Length field. The format of the Data field is determined by the code field.

Regardless of which Configuration Options are enabled, all LCP packets are always sent in the full, standard form, as if no Configuration Options were enabled. This ensures that LCP Configure-Request packets are always recognizable even when one end of the link mistakenly believes the link to be Open.

This document describes Version 1 of the Link Control Protocol. In the interest of simplicity, there is no version field in the LCP packet. If a new version of LCP is necessary in the future, the intention is that a new Data Link Layer Protocol field value should be used to differentiate Version 1 LCP from all other versions. A correctly functioning Version 1 LCP implementation will always respond to unknown Protocols (including other versions) with an easily recognizable Version 1 packet, thus providing a deterministic fallback mechanism for implementations of other versions. 


\subsubsection{Configure-Request}

Description

A LCP implementation wishing to open a connection MUST transmit a LCP packet with the Code field set to 1 (Configure-Request) and the Options field filled with any desired changes to the default link Configuration Options.

Upon reception of a Configure-Request, an appropriate reply MUST be transmitted.

A summary of the Configure-Request packet format is shown below. The fields are transmitted from left to right.

$\begin{array}{lllllllllllll}0 & 1 & 2 & 3\end{array}$

$\begin{array}{llllllllllllllllllllllllllllllll}0 & 1 & 2 & 3 & 4 & 5 & 6 & 7 & 8 & 9 & 0 & 1 & 2 & 3 & 4 & 5 & 6 & 7 & 8 & 9 & 0 & 1 & 2 & 3 & 4 & 5 & 6 & 7 & 8 & 9 & 0 & 1\end{array}$ +-+-+-+-+-+-+-+-+-+-+-+-+-+-+-+-+-+-+-+-+-+-+-+-+-+-t-+-+-+-+-+-+ Code $\mid$ Identifier $\mid \begin{aligned} & \text { Length } \\ & +-+-+-+-+-+-+-+-+-+-+-+-+-+-+-+-+-+-+-+-+-+-+-++-+-+-+-+-+-+-+-+-+\end{aligned}$

| Options ...

$+-+-+-+-+$

Code

1 for Configure-Request.

Identifier

The Identifier field should be changed on each transmission. On reception, the Identifier field should be copied into the Identifier field of the appropriate reply packet.

Options

The options field is variable in length and contains the list of zero or more Configuration Options that the sender desires to negotiate. All Configuration Options are always negotiated simultaneously. The format of Configuration Options is further described in a later section.

\subsubsection{Configure-Ack}

Description

If every Configuration Option received in a Configure-Request is both recognizable and acceptable, then a LCP implementation should transmit a LCP packet with the Code field set to 2 (Configure- 
Ack), the Identifier field copied from the received ConfigureRequest, and the Options field copied from the received Configure-Request. The acknowledged Configuration Options MUST NOT be reordered or modified in any way .

On reception of a Configure-Ack, the Identifier field must match that of the last transmitted Configure-Request, or the packet is invalid. Additionally, the Configuration Options in a ConfigureAck must match those of the last transmitted Configure-Request, or the packet is invalid. Invalid packets should be silently discarded.

Reception of a valid Configure-Ack indicates that all Configuration Options sent in the last Configure-Request are acceptable.

A summary of the Configure-Ack packet format is shown below. The fields are transmitted from left to right.

$\begin{array}{lllllllllll}0 & 1 & 2 & 3\end{array}$

$\begin{array}{llllllllllllllllllllllllllllllll}0 & 1 & 2 & 3 & 4 & 5 & 6 & 7 & 8 & 9 & 0 & 1 & 2 & 3 & 4 & 5 & 6 & 7 & 8 & 9 & 0 & 1 & 2 & 3 & 4 & 5 & 6 & 7 & 8 & 9 & 0 & 1\end{array}$

+-+-+-+-+-+-+-+-+-+-+-+-+-+-+-+-+-+-+-+-+-+-+-+-+-+-+-t-+-+-+-+-+

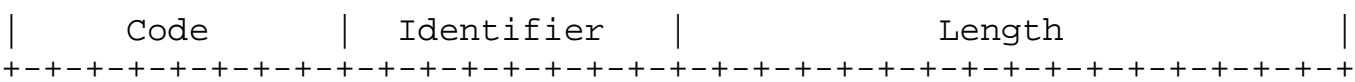

| Options ...

$+-+-+-+-+$

Code

2 for Configure-Ack.

Identifier

The Identifier field is a copy of the Identifier field of the Configure-Request which caused this Configure-Ack.

Options

The Options field is variable in length and contains the list of zero or more Configuration Options that the sender is acknowledging. All Configuration Options are always acknowledged simultaneously.

4.3.3. Configure-Nak

Description

If every element of the received Configuration Options is 
recognizable but some are not acceptable, then a LCP

implementation should transmit a LCP packet with the Code field set to 3 (Configure-Nak), the Identifier field copied from the received Configure-Request, and the Options field filled with only the unacceptable Configuration Options from the Configure-Request. All acceptable Configuration Options should be filtered out of the Configure-Nak, but otherwise the Configuration Options from the Configure-Request MUST NOT be reordered. Each of the nak'd Configuration Options MUST be modified to a value acceptable to the Configure-Nak sender. Finally, an implementation may be configured to require the negotiation of a specific option. If that option is not listed, then that option may be appended to the list of nak'd Configuration Options in order to request the remote end to list that option in its next Configure-Request packet. The appended option must include a value acceptable to the ConfigureNak sender.

On reception of a Configure-Nak, the Identifier field must match that of the last transmitted Configure-Request, or the packet is invalid and should be silently discarded.

Reception of a valid Configure-Nak indicates that a new Configure-Request should be sent with the Configuration Options modified as specified in the Configure-Nak.

A summary of the Configure-Nak packet format is shown below. The fields are transmitted from left to right.

$\begin{array}{lllllllllll}0 & 1 & 2 & 3\end{array}$

$\begin{array}{llllllllllllllllllllllllllllllll}0 & 1 & 2 & 3 & 4 & 5 & 6 & 7 & 8 & 9 & 0 & 1 & 2 & 3 & 4 & 5 & 6 & 7 & 8 & 9 & 0 & 1 & 2 & 3 & 4 & 5 & 6 & 7 & 8 & 9 & 0 & 1\end{array}$

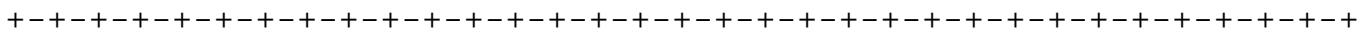
Code $\mid$ Identifier | Length
+-+-+-+-+-+-+-+-+-+-+-+-+-+-+-+-+-+-+-+-+-+-+-+-+-+-+-+-+-+-+-+-+
Options ... $+-+-+-+-+$

Code

3 for Configure-Nak.

Identifier

The Identifier field is a copy of the Identifier field of the Configure-Request which caused this Configure-Nak.

Options

The Options field is variable in length and contains the list of 
zero or more Configuration Options that the sender is nak'ing. All Configuration Options are always nak'd simultaneously.

\subsubsection{Configure-Reject}

Description

If some Configuration Options received in a Configure-Request are not recognizable or are not acceptable for negotiation (as configured by a network manager), then a LCP implementation should transmit a LCP packet with the Code field set to 4 (ConfigureReject), the Identifier field copied from the received ConfigureRequest, and the Options field filled with only the unrecognized Configuration Options from the Configure-Request. All recognizable and negotiable Configuration Options must be filtered out of the Configure-Reject, but otherwise the Configuration Options MUST not be reordered.

On reception of a Configure-Reject, the Identifier field must match that of the last transmitted Configure-Request, or the packet is invalid. Additionally, the Configuration options in a Configure-Reject must be a proper subset of those in the last transmitted Configure-Request, or the packet is invalid. Invalid packets should be silently discarded.

Reception of a Configure-Reject indicates that a new ConfigureRequest should be sent which does not include any of the Configuration Options listed in the Configure-Reject.

A summary of the Configure-Reject packet format is shown below. The fields are transmitted from left to right.

$\begin{array}{lllllllllllllll}0 & 1 & 2 & 3\end{array}$

$\begin{array}{llllllllllllllllllllllllllllllll}0 & 1 & 2 & 3 & 4 & 5 & 6 & 7 & 8 & 9 & 0 & 1 & 2 & 3 & 4 & 5 & 6 & 7 & 8 & 9 & 0 & 1 & 2 & 3 & 4 & 5 & 6 & 7 & 8 & 9 & 0 & 1\end{array}$

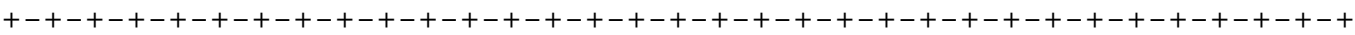
Code $\mid$ Identifier $\mid$\begin{tabular}{l|} 
Length \\
+-+-+-+-+-+-+-+-+-+-+-+-+-+-+-+-+-+-+-+-+-+-+-++-+-+-+-+-+-+-+-+-+
\end{tabular} | Options ... $+-+-+-+-+$

Code

4 for Configure-Reject.

Identifier

The Identifier field is a copy of the Identifier field of the Configure-Request which caused this Configure-Reject. 
Options

The Options field is variable in length and contains the list of zero or more Configuration Options that the sender is rejecting. All Configuration Options are always rejected simultaneously.

4.3.5. Terminate-Request and Terminate-Ack

Description

LCP includes Terminate-Request and Terminate-Ack Codes in order to provide a mechanism for closing a connection.

A LCP implementation wishing to close a connection should transmit a LCP packet with the Code field set to 5 (Terminate-Request) and the Data field filled with any desired data. Terminate-Request packets should continue to be sent until Terminate-Ack is received, the Physical Layer indicates that it has gone down, or a sufficiently large number have been transmitted such that the remote end is down with reasonable certainty.

Upon reception of a Terminate-Request, a LCP packet MUST be transmitted with the code field set to 6 (Terminate-Ack), the Identifier field copied from the Terminate-Request packet, and the Data field filled with any desired data.

Reception of an unelicited Terminate-Ack indicates that the connection has been closed.

A summary of the Terminate-Request and Terminate-Ack packet formats is shown below. The fields are transmitted from left to right.

$\begin{array}{lllllllllllllllll}0 & 1 & 2 & 3\end{array}$

$\begin{array}{llllllllllllllllllllllllllllllll}0 & 1 & 2 & 3 & 4 & 5 & 6 & 7 & 8 & 9 & 0 & 1 & 2 & 3 & 4 & 5 & 6 & 7 & 8 & 9 & 0 & 1 & 2 & 3 & 4 & 5 & 6 & 7 & 8 & 9 & 0 & 1\end{array}$

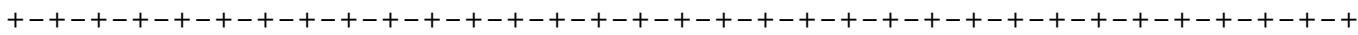
\begin{tabular}{|c|c|} 
Code & Identifier | Iength \\
+-+-+-+-+-+-+-+-+-+-+-+-+-+-+-+-+-+-+-+-+-+-+-+-+-+-+-+-+-+-+-+-+ \\
Data..
\end{tabular}

Code

5 for Terminate-Request;

6 for Terminate-Ack. 


\section{Identifier}

The Identifier field is one octet and aids in matching requests and replies.

Data

The Data field is zero or more octets and contains uninterpreted data for use by the sender. The data may consist of any binary value and may be of any length from zero to the established value for the peer's MRU.

\subsubsection{Code-Reject}

Description

Reception of a LCP packet with an unknown Code indicates that one of the communicating LCP implementations is faulty or incomplete. This error MUST be reported back to the sender of the unknown Code by transmitting a LCP packet with the Code field set to 7 (CodeReject), and the inducing packet copied to the Rejected-Packet field.

Upon reception of a Code-Reject, a LCP implementation should make an immediate transition to the closed state, and should report the error, since it is unlikely that the situation can be rectified automatically.

A summary of the Code-Reject packet format is shown below. The fields are transmitted from left to right.

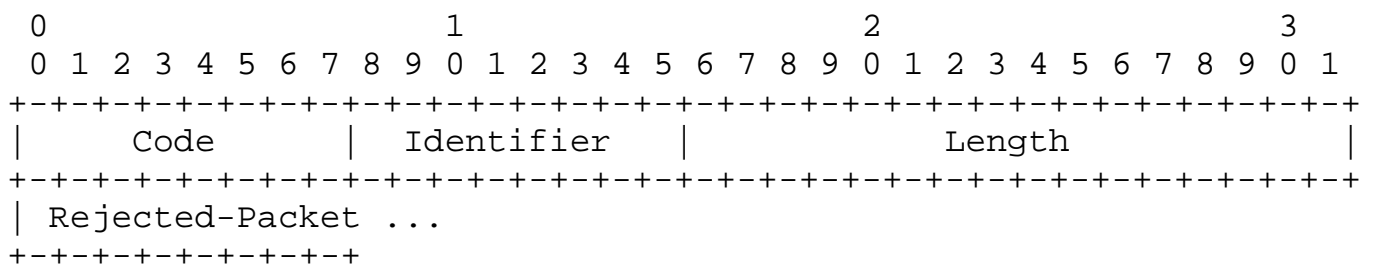

Code

7 for Code-Reject.

Identifier

The Identifier field is one octet and is for use by the transmitter. 
Rejected-Packet

The Rejected-Packet field contains a copy of the LCP packet which is being rejected. It begins with the rejected code field; it does not include any PPP Data Link Layer headers. The RejectedPacket should be truncated to comply with the established value of the peer's MRU.

\subsubsection{Protocol-Reject}

\section{Description}

Reception of a PPP frame with an unknown Data Link Layer Protocol indicates that the remote end is attempting to use a protocol which is unsupported at the local end. This typically occurs when the remote end attempts to configure a new, but unsupported protocol. If the LCP state machine is in the Open state, then this error MUST be reported back to the sender of the unknown protocol by transmitting a LCP packet with the Code field set to 8 (Protocol-Reject), the Rejected-Protocol field set to the received Protocol, and the Data field filled with any desired data.

Upon reception of a Protocol-Reject, a LCP implementation should stop transmitting frames of the indicated protocol.

Protocol-Reject packets may only be sent in the LCP Open state. Protocol-Reject packets received in any state other than the LCP Open state should be discarded and no further action should be taken.

A summary of the Protocol-Reject packet format is shown below. The fields are transmitted from left to right.

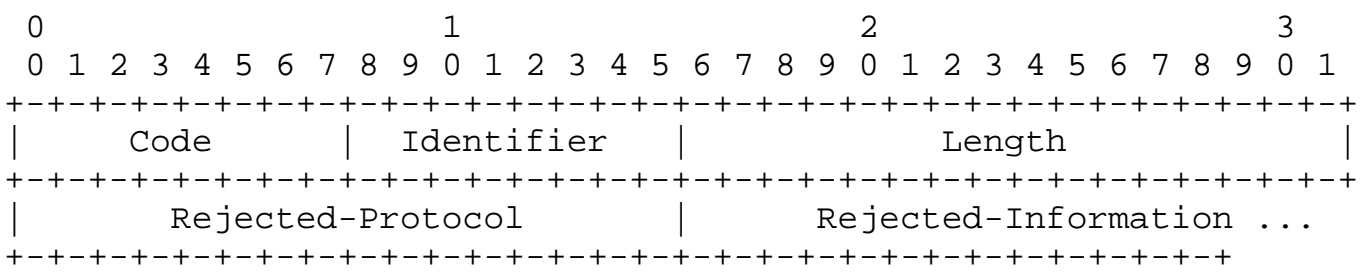

Code

8 for Protocol-Reject.

Identifier

The Identifier field is one octet and is for use by the 
transmitter.

Rejected-Protocol

The Rejected-Protocol field is two octets and contains the Protocol of the Data Link Layer frame which is being rejected.

Rejected-Information

The Rejected-Information field contains a copy from the frame which is being rejected. It begins with the Information field, and does not include any PPP Data Link Layer headers or the FCS. The Rejected-Information field should be truncated to comply with the established value of the peer's MRU.

\subsubsection{Echo-Request and Echo-Reply}

Description

LCP includes Echo-Request and Echo-Reply Codes in order to provide a Data Link Layer loopback mechanism for use in exercising both directions of the link. This is useful as an aid in debugging, link quality determination, performance testing, and for numerous other functions.

An Echo-Request sender transmits a LCP packet with the Code field set to 9 (Echo-Request) and the Data field filled with any desired data, up to but not exceeding the receivers established MRU.

Upon reception of an Echo-Request, a LCP packet MUST be transmitted with the Code field set to 10 (Echo-Reply), the Identifier field copied from the received Echo-Request, and the Data field copied from the Echo-Request, truncating as necessary to avoid exceeding the peer's established MRU.

Echo-Request and Echo-Reply packets may only be sent in the LCP Open state. Echo-Request and Echo-Reply packets received in any state other than the LCP Open state should be discarded and no further action should be taken.

A summary of the Echo-Request and Echo-Reply packet formats is shown below. The fields are transmitted from left to right. 


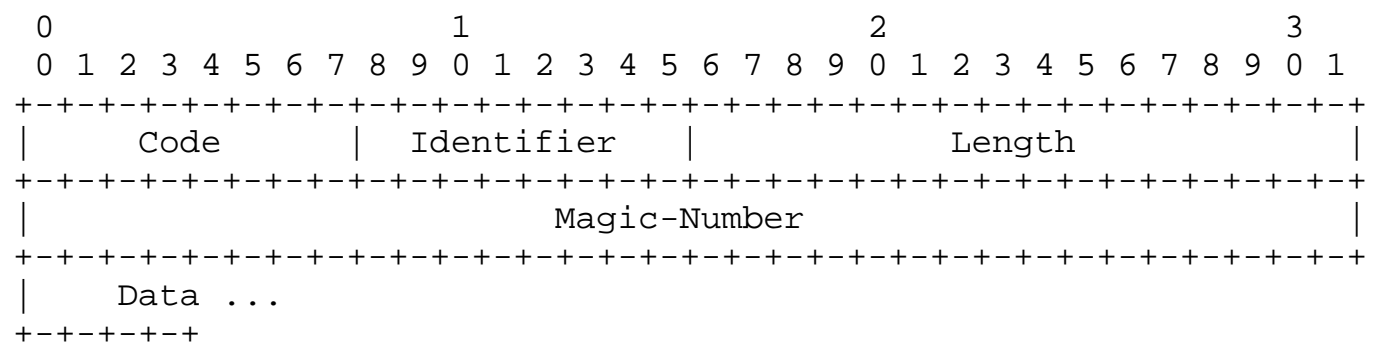

Code

9 for Echo-Request;

10 for Echo-Reply.

Identifier

The Identifier field is one octet and aids in matching EchoRequests and Echo-Replies.

Magic-Number

The Magic-Number field is four octets and aids in detecting loopbacked links. Unless modified by a Configuration Option, the Magic-Number MUST always be transmitted as zero and MUST always be ignored on reception. Further use of the Magic-Number is beyond the scope of this discussion.

Data

The Data field is zero or more octets and contains uninterpreted data for use by the sender. The data may consist of any binary value and may be of any length from zero to the established value for the peer's MRU.

\subsubsection{Discard-Request}

Description

LCP includes a Discard-Request Code in order to provide a Data Link Layer data sink mechanism for use in exercising the local to remote direction of the link. This is useful as an aid in debugging, performance testing, and and for numerous other functions.

A discard sender transmits a LCP packet with the Code field set to 11 (Discard-Request) and the Data field filled with any desired 
data, up to but not exceeding the receivers established MRU.

A discard receiver MUST simply throw away an Discard-Request that it receives.

Discard-Request packets may only be sent in the LCP Open state.

A summary of the Discard-Request packet formats is shown below. The fields are transmitted from left to right.

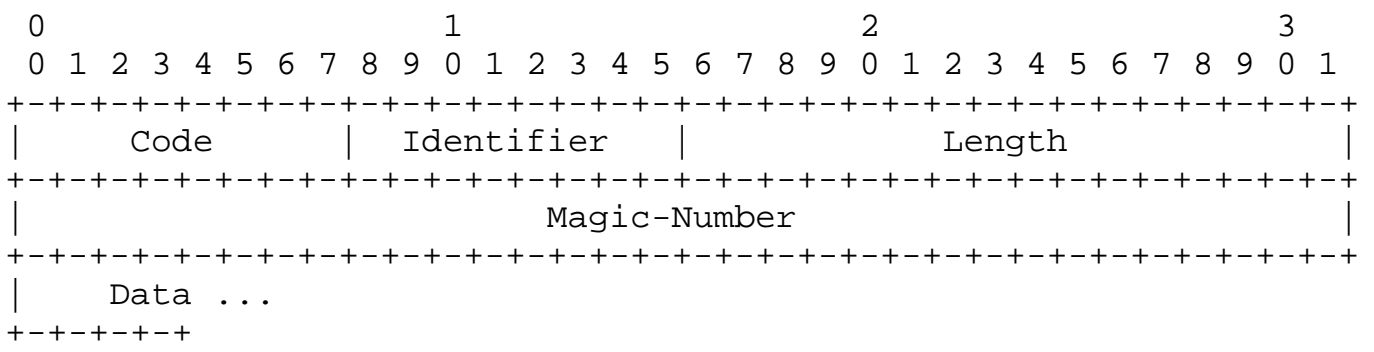

Code

11 for Discard-Request.

Identifier

The Identifier field is one octet and is for use by the DiscardRequest transmitter.

Magic-Number

The Magic-Number field is four octets and aids in detecting loopbacked links. Unless modified by a configuration option, the Magic-Number MUST always be transmitted as zero and MUST always be ignored on reception. Further use of the Magic-Number is beyond the scope of this discussion.

Data

The Data field is zero or more octets and contains uninterpreted data for use by the sender. The data may consist of any binary value and may be of any length from zero to the established value for the peer's MRU.

\subsection{Configuration Options}

LCP Configuration Options allow modifications to the standard characteristics of a point-to-point link to be negotiated. 
Negotiable modifications include such things as the maximum receive unit, async control character mapping, the link authentication method, the link encryption method, etc.. The Configuration Options themselves are described in separate documents. If a Configuration Option is not included in a Configure-Request packet, the default value for that Configuration Option is assumed.

The end of the list of Configuration Options is indicated by the end of the LCP packet.

Unless otherwise specified, a specific Configuration Options should be listed no more than once in a Configuration Options list. Specific Configuration Options may override this general rule and may be listed more than once. The effect of this is Configuration Option specific and is specified by each such Configuration option.

Also unless otherwise specified, all Configuration options apply in a half-duplex fashion. When negotiated, they apply to only one direction of the link, typically in the receive direction when interpreted from the point of view of the Configure-Request sender.

4.4.1. Format

A summary of the Configuration Option format is shown below. The fields are transmitted from left to right.

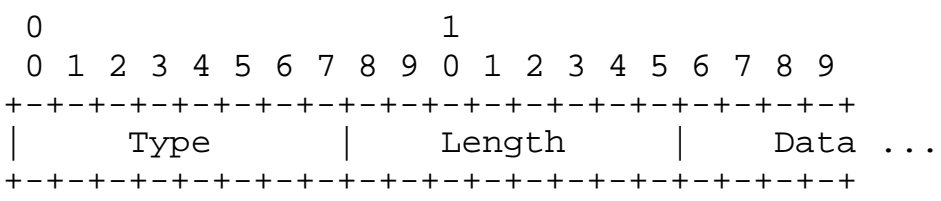

Type

The Type field is one octet and indicates the type of Configuration Option. The most up-to-date values of the Type field are specified in the most recent "Assigned Numbers" RFC [11].

Length

The Length field is one octet and indicates the length of this Configuration Option including the Type, Length and Data fields. If a negotiable Configuration option is received in a ConfigureRequest but with an invalid Length, a Configure-Nak should be transmitted which includes the desired Configuration Option with an appropriate Length and Data. 
Data

The Data field is zero or more octets and indicates the value or other information for this Configuration Option. The format and length of the Data field is determined by the Type and Length fields.

5. A PPP Network Control Protocol (NCP) for IP

The IP Control Protocol (IPCP) is responsible for configuring, enabling, and disabling the IP protocol modules on both ends of the point-to-point link. As with the Link Control Protocol, this is accomplished through an exchange of packets. IPCP packets may not be exchanged until LCP has reached the network-layer Protocol Configuration Negotiation phase. Likewise, IP datagrams may not be exchanged until IPCP has first opened the connection.

The IP Control Protocol is exactly the same as the Link Control Protocol with the following exceptions:

Data Link Layer Protocol Field

Exactly one IP Control Protocol packet is encapsulated in the Information field of PPP Data Link Layer frames where the Protocol field indicates type hex 8021 (IP Control Protocol).

Code field

Only Codes 1 through 7 (Configure-Request, Configure-Ack, Configure-Nak, Configure-Reject, Terminate-Request, Terminate-Ack and Code-Reject) are used. Other Codes should be treated as unrecognized and should result in Code-Rejects.

Timeouts

IPCP packets may not be exchanged until the Link Control Protocol has reached the network-layer Protocol Configuration Negotiation phase. An implementation should be prepared to wait for Link Quality testing to finish before timing out waiting for a Configure-Ack or other response. It is suggested that an implementation give up only after user intervention or a configurable amount of time.

Configuration Option Types

The IPCP has a separate set of Configuration Options. The most up-to-date values of the type field are specified in the most recent "Assigned Numbers" RFC [11]. 


\subsection{Sending IP Datagrams}

Before any IP packets may be communicated, both the Link Control Protocol and the IP Control Protocol must reach the open state.

Exactly one IP packet is encapsulated in the Information field of PPP Data Link Layer frames where the Protocol field indicates type hex 0021 (Internet Protocol).

The maximum length of an IP packet transmitted over a PPP link is the same as the maximum length of the Information field of a PPP data link layer frame. Larger IP datagrams must be fragmented as necessary. If a system wishes to avoid fragmentation and reassembly, it should use the TCP Maximum segment Size option [12], or a similar mechanism, to discourage others from sending large datagrams.

\section{A. Asynchronous HDLC}

This appendix summarizes the modifications to ISO 3309-1979 proposed in ISO 3309:1984/PDAD1. These modifications allow HDLC to be used with 8-bit asynchronous links.

Transmission Considerations

Each octet is delimited by a start and a stop element.

Flag Sequence

The Flag Sequence is a single octet and indicates the beginning or end of a frame. The Flag Sequence consists of the binary sequence 01111110 (hexadecimal 0x7e).

Transparency

On asynchronous links, a character stuffing procedure is used. The Control Escape octet is defined as binary 01111101 (hexadecimal $0 \times 7 \mathrm{~d}$ ) where the bit positions are numbered 87654321 (not 76543210, BEWARE).

After FCS computation, the transmitter examines the entire frame between the two Flag Sequences. Each Flag Sequence, Control Escape octet and octet with value less than hexadecimal 0x20 is replaced by a two character sequence consisting of the Control Escape octet and the original octet with bit 6 complemented (i.e., exclusive-or'd with hexadecimal 0x20).

Prior to FCS computation, the receiver examines the entire frame between the two Flag Sequences. For each Control Escape octet, 
that octet is removed and bit 6 of the following octet is complemented. A Control Escape octet immediately preceding the closing Flag sequence indicates an invalid frame.

Note: The inclusion of all octets less than hexadecimal $0 \times 20$ allows all ASCII control characters [10] excluding DEL (Delete) to be transparently communicated through almost all known data communications equipment.

A few examples may make this more clear. Packet data is transmitted on the link as follows:

$0 \times 7 e$ is encoded as $0 \times 7 d, 0 \times 5 e$.

$0 \times 7 d$ is encoded as $0 \times 7 d, 0 \times 5 d$.

$0 \times 01$ is encoded as $0 \times 7 d, 0 \times 21$.

Aborting a Transmission

On asynchronous links, frames may be aborted by transmitting a "0" stop bit where a "1" bit is expected (framing error) or by transmitting a Control Escape octet followed immediately by a closing Flag Sequence.

Inter-frame Time Fill

On asynchronous links, inter-octet and inter-frame time fill should be accomplished by transmitting continuous "1" bits (markhold state).

Note: On asynchronous links, inter-frame time fill can be viewed as extended inter-octet time fill. Doing so can save one octet for every frame, decreasing delay and increasing bandwidth. This is possible since a Flag sequence may serve as both a frame close and a frame begin. After having received any frame, an idle receiver will always be in a frame begin state.

Robust transmitters should avoid using this trick overzealously since the price for decreased delay is decreased reliability. Noisy links may cause the receiver to receive garbage characters and interpret them as part of an incoming frame. If the transmitter does not transmit a new opening Flag Sequence before sending the next frame, then that frame will be appended to the noise characters causing an invalid frame (with high reliability). Transmitters should avoid this by transmitting an open Flag Sequence whenever "appreciable time" has elapsed since the prior closing Flag sequence. It is suggested that implementations will achieve the best results by 
always sending an opening Flag sequence if the new frame is not back-to-back with the last. The maximum value for "appreciable time" is likely to be no greater than the typing rate of a slow to average typist, say 1 second.

B. Fast Frame Check Sequence (FCS) Implementation

\section{B.1. FCS Computation Method}

The following code provides a table lookup computation for calculating the Frame Check Sequence as data arrives at the interface. The table is created by the code in section 2 .

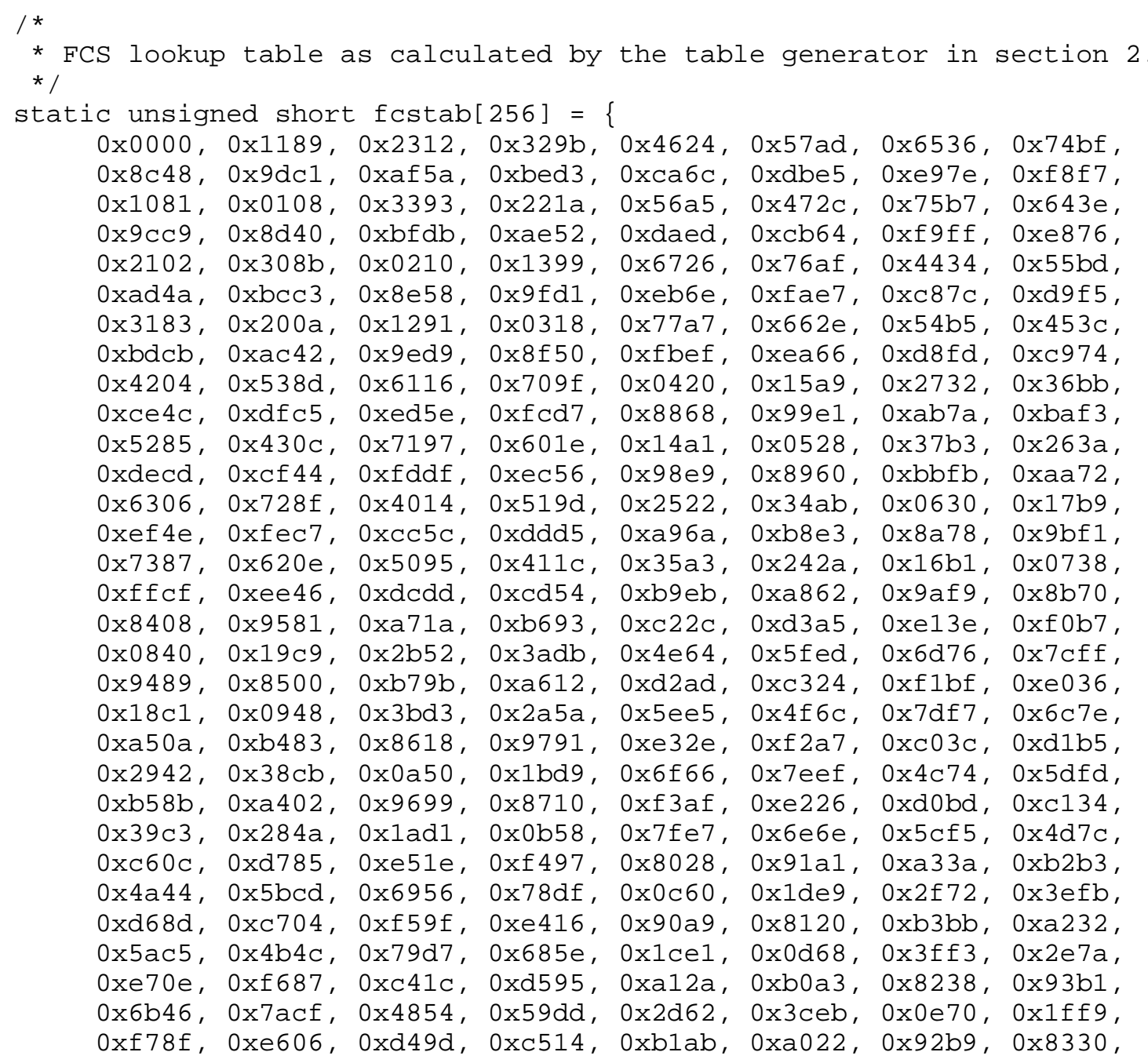




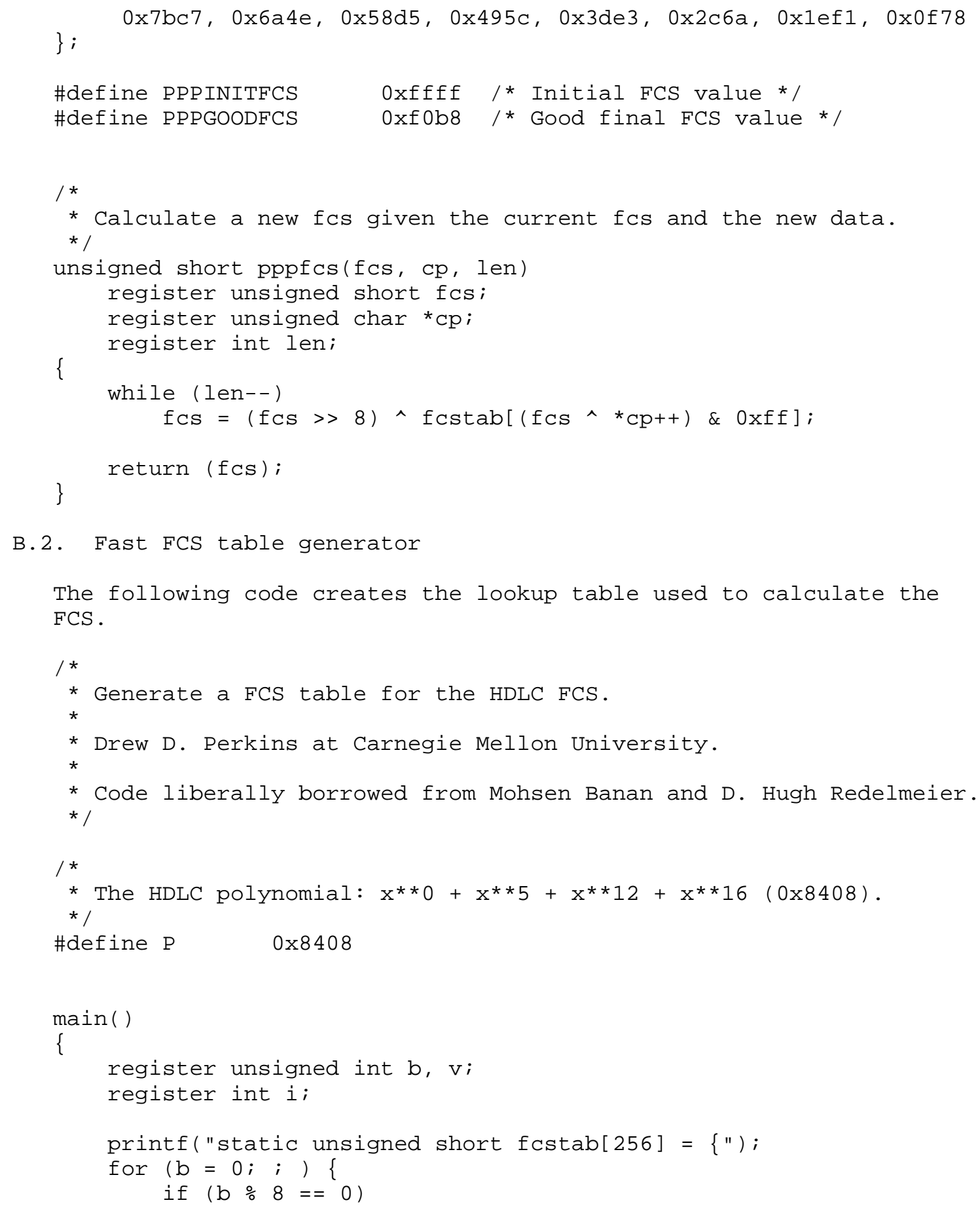




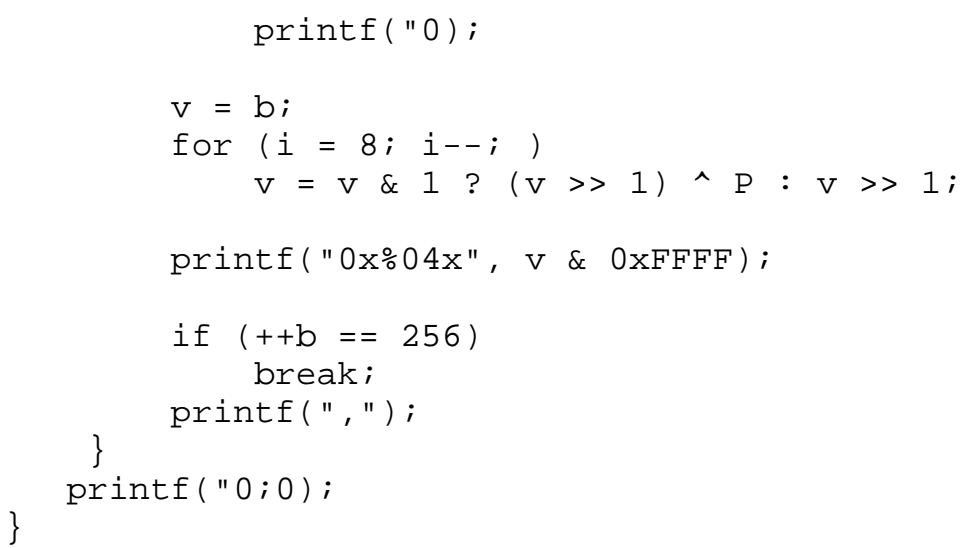

\section{References}

[1] Electronic Industries Association, "Interface Between Data Terminal Equipment and Data Communications Equipment Employing Serial Binary Data Interchange", EIA Standard RS-232-C, August 1969 .

[2] International Organization For Standardization, "Data Communication - High-level Data Link Control Procedures - Frame Structure", ISO Standard 3309-1979, 1979.

[3] International Organization For Standardization, "Data Communication - High-level Data Link Control Procedures Elements of Procedures", ISO Standard 4335-1979, 1979.

[4] International Organization For Standardization, "Data Communication - High-Level Data Link Control Procedures Elements of Procedures - Addendum 1", ISO Standard 43351979 /Addendum 1, 1979.

[5] International Organization For Standardization, "Information Processing Systems - Data Communication - High-level Data Link Control Procedures - Frame structure - Addendum 1: Start/stop Transmission", Proposed Draft International Standard ISO 3309:1983/PDAD1, 1984 .

[6] International Telecommunication Union, CCITT Recommendation X.25, "Interface Between Data Terminal Equipment (DTE) and Data Circuit Terminating Equipment (DCE) for Terminals Operating in the Packet Mode on Public Data Networks", CCITT Red Book, Volume VIII, Fascicle VIII.3, Rec. X.25., October 1984. 
[7] Perez, "Byte-wise CRC Calculations", IEEE Micro, June 1983. Morse, G., "Calculating CRC's by Bits and Bytes", Byte, September 1986 .

[8] LeVan, J., "A Fast CRC", Byte, November 1987.

[9] American National Standards Institute, "American National Standard Code for Information Interchange", ANSI X3.4-1977, 1977.

[10] Postel, J., "Internet Protocol", RFC 791, USC/Information Sciences Institute, September 1981.

[11] Reynolds, J.K., and J. Postel, "Assigned Numbers", RFC 1010, USC/Information Sciences Institute, May 1987.

[12] Postel, J., "The TCP Maximum Segment Size Option and Related Topics", RFC 879, USC/Information Sciences Institute, November 1983.

Security Considerations

Security issues are not addressed in this memo.

Author's Address

This proposal is the product of the Point-to-Point Protocol Working Group of the Internet Engineering Task Force (IETF). The working group can be contacted via the chair:

Russ Hobby

UC Davis

Computing Services

Davis, CA 95616

Phone: (916) 752-0236

EMail: rdhobby@ucdavis.edu

Acknowledgments

Many people spent significant time helping to develop the Point-toPoint Protocol. The complete list of people is too numerous to list, but the following people deserve special thanks: Ken Adelman (TGV), Craig Fox (NSC), Phill Gross (NRI), Russ Hobby (UC Davis), David Kaufman (Proteon), John LoVerso (Xylogics), Bill Melohn (Sun Microsystems), Mike Patton (MIT), Drew Perkins (CMU), Greg Satz (cisco systems) and Asher Waldfogel (Wellfleet). 